\title{
Suppression of optokinesis by a stabilized target: Effects of instruction and stimulus frequency
}

\author{
JORDAN POLA, HARRY J. WYATT, and MARCIE LUSTGARTEN \\ State University of New York, New York, New York
}

\begin{abstract}
Subjects viewed a foveally stabilized target presented against a background field of dots moving sinusoidally. Several different modes of viewing the target were used (subjects were instructed to gaze, look, or hold), and the frequency of sinusoidal field motion was varied from 1/32 to $2 \mathrm{~Hz}$. In line with previous findings, the presence of a stabilized target resulted in substantial suppression of optokinesis. The characteristics of this suppression (gain and phase of slow residual eye movements) were dependent on both the mode of viewing the target and the frequency of field motion. When subjects used an imaginary target, little suppression occurred. These findings provide an overall profile of dynamic characteristics of mechanisms involved in the suppression of optokinesis. They support the view that this suppression is significantly determined by the presence of a target against a moving background (even without retinal slip), and by the mode of attending to the target.
\end{abstract}

The optokinetic system is responsible for visual stabilization of gaze relative to the whole visual field. For animals with centralized retinas, however, it is often important to fixate targets in the presence of relative motion of the visual world, and in such cases, optokinesis must be suppressed. Many aspects of such suppression remain unclear. One possibility is that suppression is accomplished by an oculomotor system (e.g., such as the smooth pursuit system) using retinal slip of a foveal target to drive the eyes opposite to the motion of the optokinetic field (thus nulling the optokinesis). Recent studies of this issue (Murasugi, Howard, \& Ohmi, 1986, 1989) have examined how stationary contours (bars) can be used to stabilize gaze against optokinesis: to be effective, bars had to be perpendicular to (or at least not parallel to) field motion. (Vertical bars were more effective in suppressing horizontal $\mathrm{OKN}$ than horizontal bars were.) Bars were most effective when close to the fovea, which is consistent with bar retinal slip being involved in suppression. However, besides configuration and location of the bars, the amount of suppression depended on whether subjects attended to the bars or not. Suppression was greatest during attention to the bars and least during attention to the optokinetic field.

Although these results reveal important characteristics of the suppression of optokinesis, a possible limiting feature of the studies is that they were conducted in the

This research was supported by NSF Grant BNS 85-19267 and the Schnurmacher Institute for Vision Research; the authors are in the Schnurmacher Institute, which is part of the State College of Optometry. The authors thank John Orzuchowski for help with electronics and Max Brinson for help with the figures. Correspondence concerning this article should be sent to Jordan Pola, Schnurmacher Institute for Vision Research, State University of New York, State College of Optometry, 100 East 24th Street, New York, NY 10010. closed-loop condition; eye movements could eliminate differences between eye and target (bar) position and velocity. Certain questions are difficult to answer in such conditions; in particular, it is hard to determine the extent to which mechanisms responsible for suppression make use of target retinal slip (a use that may be influenced by instructions), as compared with, for example, the relative motion of target and field.

One way to separate these contributions is to stabilize the target at the retina, eliminating target retinal motion (but leaving field retinal motion and especially target-field relative motion). This is related to a technique used to investigate the sensitivity of the peripheral retina to optokinetic stimuli, in which a large blank zone, stabilized and centered on the fovea, is superimposed on an optokinetic field so that only the peripheral retina is stimulated optokinetically (Cheng \& Outerbridge, 1975; Dubois \& Collewijn, 1979; Gresty \& Halmagyi, 1979; Koerner \& Schiller, 1972; van Die \& Collewijn, 1982). This stimulus typically generates weak optokinesis, which supports the idea that the peripheral retina provides weak input to the optokinetic system. However, such results are confounded by the fact that the blank zone has boundaries perpendicular to field motion, which may be partly responsible for the weak optokinesis (Murasugi et al., 1986, 1989). A further difficulty in interpreting most of these studies is that there is little specification of instructions to subjects with respect to attending to the blank zone and/or to the optokinetic field.

In our initial investigation of suppression of optokinesis (Wyatt \& Pola, 1984), we asked subjects to actively look at a small round $\left(1.5^{\circ}\right)$ target stabilized at the fovea, presented against the sinusoidal motion of an optokinetic field. Clear suppression of optokinesis was observed, even though retinal slip of the foveal target was absent. In addition, almost all of the subjects showed residual slow eye 
movements with large phase lags relative to (in many cases roughly in counterphase with) the field motion. In a second study (Wyatt, Pola, \& Lustgarten, 1988), we asked subjects to simply gaze ahead, avoiding deliberate regard of either the stabilized target or the moving field; substantial suppression of optokinesis still occurred, even with eccentrically stabilized targets, although the suppression was less marked than it was in the previous study. These investigations indicated that target retinal slip was not essential for the suppression of optokinesis; moreover, since relative target-field motion was present (though target retinal slip was absent), this relative motion was a likely candidate as a stimulus for suppression. The results also clearly imply the ability of instructions to the subjects to modulate the behavior. Similar results and interpretations have since been reported by Howard, Giaschi, and Murasugi (1989).

A significant feature of these findings is that the onset of suppression is quite rapid, occurring within about 100-200 msec (Wyatt \& Pola, 1984), suggesting the existence of a well-developed, relatively automatic control process. However, little is known about this process except for its behavior with 0.25 - and $0.5-\mathrm{Hz}$ sinusoidal field motion (Wyatt \& Pola, 1984; Wyatt et al., 1988) and unidirectional field motion (Howard et al., 1989). In the present study, we attempted to determine the overall dynamic properties of the suppression mechanism. In addition to employing conditions similar to those noted above, we also investigated the effects of deliberate subject effort on suppression in two further conditions, which we call the hold and hold-no-target conditions (see Method section).

Portions of these results were presented in preliminary form at the 1987 annual meetings of the Association for Research in Vision and Ophthalmology in Sarasota, FL, and the Society for Neuroscience in New Orleans, LA.

\section{METHOD}

\section{Visual Stimuli}

The subjects sat in a dark room (painted black, with only the visual stimuli visible) and viewed the stimuli monocularly with the left eye, using a bite bar made from dental impression compound to stabilize head position. In each trial, the subjects observed a round target (stabilized on the fovea) presented against an optokinetic stimulus field (not stabilized), or the field alone. The target $\left(4^{\circ}\right.$ diam; $\left.0.5 \mathrm{~cd} / \mathrm{m}^{2}\right)$ was rear-projected on a screen via a servomotorcontrolled mirror (General Scanning), and it was stabilized on the fovea with respect to horizontal eye movements by using a signal of horizontal eye position. This stabilization signal had small amounts of noise and drift (see under Measurement of Eye Position, below). However, perfect stabilization would not be desirable, since target disappearance would interfere with the general purpose of the experiment. The servomotor/mirror system has a flat gain and little phase lag at frequencies involved in eye movements; when driven by the eye-position signal from a saccade, the mirror-position signal replicated the saccade signal almost exactly (thus, the system could not distort the oculomotor response in these studies). We used the relatively large target to reduce any instabilities of fixation of the stabilized target that might be due to small drifts in the eye-position signal (i.e., offset of a stabilized target elicits eye movements toward the target; see Pola \& Wyatt, 1980).
The optokinetic stimulus was created with a planetarium projectora drum with small holes drilled in it and a compact-filament source at the center. Pinhole images of the filament were formed on the walls, ceiling, and floor (the diameter of these images was approximately $2^{\circ}$ ). A servomotor (Electrocraft) rotated the drum, producing horizontal sinusoidal field motion (for further details, see Wyatt \& Pola, 1988). The maximum field velocity was kept constant at $15.7^{\circ} / \mathrm{sec}$; the frequency varied from $1 / 32$ to $2 \mathrm{~Hz}$ in octaves.

\section{Measurement of Eye Position}

We used an infrared reflection system (Eye Trac Model 200, Narco Bio-Systems), modified to reduce noise and to permit electronic adjustment of the left/right balance of the infrared sensors. Records run at high gain showed microsaccades and drifts, and the level of high-frequency noise was small $(<2-3 \mathrm{~min}$ arc). The level of low-frequency noise, estimated from drift during a 30- to 40sec fixation trial, could be kept to about $10 \mathrm{~min}$ arc or less during the trial (some of which could have been actual fixation drift). After careful adjustment, the system was linear to several parts in a hundred over a range of about $\pm 20^{\circ}$.

\section{Data Recording and Analysis}

Horizontal eye position was filtered (passive single-stage lowpass filter; corner $125 \mathrm{~Hz}$ ), A/D converted, and recorded on disk at $100 \mathrm{samples} / \mathrm{sec}$. This sampling rate is appropriate for signals below $50 \mathrm{~Hz}$, which is adequate because the smooth eye-movement power spectrum extends up to about $5-10 \mathrm{~Hz}$ or less (Bahill \& McDonald, 1983). The main contaminant in the signal (aside from some inevitable $60-\mathrm{Hz}$ noise) was a signal with a $2-\mathrm{kHz}$ fundamental, arising from chopping circuitry in the eye-position measurement system. The $125-\mathrm{Hz}$ filter was chosen at about the (geometric) mean of $2 \mathrm{kHz}$ and frequencies of interest; this substantially attenuated the $2-\mathrm{kHz}$ signal while only minimally shifting phase in the domain of interest. We also recorded stimulus velocity and eye position and velocity on a Grass polygraph (BW 0-75 Hz).

During analysis, an eye-position record was treated as a series of individual response cycles; average eye position and peak-topeak amplitude were calculated for each cycle. Before the cycles were included in the analyzed response, they were tested against the following criteria: (1) average eye position $<5^{\circ}$ from straight ahead, and (2) deviation of cycle amplitude from the mean for the trial $<1.5 \times$ the standard deviation for the trial. To restrict consideration to fairly steady trials, we also required the standard deviation for the trial to be $<0.5 \times$ the average amplitude. At frequencies above $0.125 \mathrm{~Hz}$, we routinely discarded the first response cycle.

For selected data segments, the position record was digitally smoothed (10-Hz corner) and differentiated. Saccades and blinks were detected and deleted by an interactive program, and replaced by straight-line segments. (We routinely use "jerk" to detect saccades; the third derivative of eye position is usually a good indicator of saccade initiation, even for small saccades in the presence of large smooth movements, when velocity and acceleration are not reliable.) Cycles substantially distorted by blinks were discarded. The cycles were then averaged, and the Fourier fundamental was calculated. Gain and phase lag of the fundamental (smooth eye velocity with respect to field velocity) were used as response values for the trial; each mean value plotted is the mean of three to five such single-trial values.

The data selection process described above usually rejected only a few cycles, since responses were quite stable. The exception was the look condition (described below). In this condition, subjects looked at the stabilized target, and responses occasionally became unstable: a small drift in eye-position signal produces a target offset, and an offset stabilized target produces a vigorous smooth eye movement (Pola \& Wyatt, 1980). To see how much effect the selection process had on the results, we analyzed some look condition trials both with and without data selection; the average values were quite similar. 


\section{Instructions to Subjects}

The study involved five types of trials: one consisted of optokinetic eye movements to the field alone (the passive condition), and the other four were concerned with suppression of optokinesis following different instructions for regarding the target. The subjects were given detailed instructions about the different tasks before beginning the experiment, and they were briefly reminded about what to do before each trial.

\section{Optokinetic Condition: Passive}

No target was present, and the subjects were to avoid deliberate fixation of any part of the optokinetic stimulus, but were instead to passively stare ahead; however, they were not to fight any involuntary eye movement that they became aware of. They were to keep their attention at the plane of the wall in front of them, and not gaze into some imagined distance or at an imaginary target. Finally, they were asked to keep their gaze roughly horizontal, which they achieved with little effort (Wyatt \& Pola, 1984).

\section{Suppression Conditions}

Gaze. This was the same as the passive condition, except that a target was present. In addition to avoiding deliberate regard of elements of the optokinetic stimulus, the subjects were also to ignore the presence of the target. We used this condition earlier to study suppression in the absence of deliberate efforts to fixate the target (Wyatt et al., 1988).

Look. The subjects were to look attentively at the target. We previously used this condition to study suppression (Wyatt \& Pola, 1984) and also to obtain smooth pursuit of a moving target in both closed-loop and open-loop conditions. The subjects were told to avoid any effort to influence target behavior in space.

Hold. The subjects attempted to hold the target directly in front of them, as stationary as possible.

Hold-no-target. No target was present, but the subjects were to create a mental image of a target fixed on the wall directly in front of them and to fixate it intently, thereby holding their eyes as stationary as possible.

\section{Experimental Protocol}

The subjects were dark adapted for $10 \mathrm{~min}$ before each session. Before each trial, the eye-position signal was calibrated by asking the subject to look at fixation lights located straight ahead and $15^{\circ}$ to the left and right of straight ahead. The foveally stabilized target was turned on during calibration; the criterion for good stabilization was that the target be seen by the experimenter to fall upon each fixation light in turn. Target centering on each fixation light could be determined to within $0.25-0.5 \mathrm{~cm}$, amounting to concentricity to within $0.12^{\circ}-0.25^{\circ}$. On a given day, trials were typically run at two frequencies for a total of 8-10 trials, each lasting 20$96 \mathrm{sec}$, plus time for calibration and rest between trials. The different conditions at a given frequency were run in quasirandom order.

\section{Subjects}

The subjects were 2 males and 3 females, 20-45 years of age; they were emmetropic to 5D myopic, uncorrected. Two of the subjects were naive.

\section{RESULTS}

\section{Passive, Gaze, and Look Conditions}

Figure 1 shows responses at several frequencies for 1 subject (S1) in the passive condition. Slow and quick phases of optokinetic nystagmus can be seen at $1 / 16,1 / 8$, and $1 / 4 \mathrm{~Hz}$. Next to each record in Figure 1 is a vector representation of the average value (over all trials) for gain and phase lag of the slow phase. The length of the arrow indicates gain, and the angle clockwise from upward indicates the phase lag of slow eye velocity behind field velocity (see the Method section). In the passive condition, at low frequencies, gain was near one and phase lag was small; as frequency increased, response amplitude fell off and phase lag increased.

Figures $2 \mathrm{~A}$ and $2 \mathrm{~B}$ show responses for $\mathrm{S} 1$ in the gaze and look conditions, again with a vector representation of average value. In the gaze condition, there were few or no quick phases and the gain of the slow responses was sharply reduced in comparison with the gain of the passive optokinetic responses (Figure 1). However, at both the low and the higher frequencies, the phase lags appear to be similar to those of the passive optokinesis. These features of the response indicate substantial suppression of optokinesis by the stabilized target, and they correspond to what we have called "passive suppression" (Wyatt et al., 1988). The responses in the look condition were quite different: both quick and slow optokinetic movements were absent, and in their place, slow movements occurred with a large phase lag relative to the field (approximately counterphase in these records). Thus, in the look condition, optokinesis was suppressed and residual movements remained. It should be noted that the look responses shown in this figure come from a subject who typically showed relatively large-amplitude residual movements, whereas many subjects had much smaller amplitudes (though similar phase lags).

Data for the 5 subjects in the passive, gaze, and look conditions are shown as a function of frequency in Figure 3. Each point in these polar plots may be regarded as the tip of a vector (as in Figures 1 and 2), showing the average value of gain and phase for the particular condition (zero phase lag, which is straight up, represents the motion of the field). The points for a given condition are connected by lines, and the frequency is marked alongside some of the points-usually for $1 / 32,1 / 4$, and $2 \mathrm{~Hz}$. The general trends described above for individual responses may be seen clearly here; thus, the curves for the passive condition (plus signs) all start at low frequencies with high gain and phase lag near zero (often with a slight lead rather than lag). As frequency increases, the curves sweep around, with gain decreasing and phase lag increasing (reaching about $90^{\circ}$ at $2 \mathrm{~Hz}$ ). In the gaze condition (circles), the gain is scaled down to notably lower values at all frequencies. However, for 4 out of the 5 subjects, phases in the gaze condition appear to be similar to those in the passive condition. (The exception, S4, is discussed below.) A two-way analysis of variance (ANOVA) performed on the phases in the two conditions indicated the existence of a significant effect of instructions $[F(1,3)=$ $18.1, p<.03]$ and an interaction between instruction and frequency $[F(6,18)=47.1, p<.02]$. However, a post hoc Tukey test showed that this could be attributed to a significant difference in phase between the conditions occurring only at $1 \mathrm{~Hz}$ [ $C$ diff $=35.5, p<.01]$. As expected, there was an effect of frequency $[F(6,18)=47.1$, 

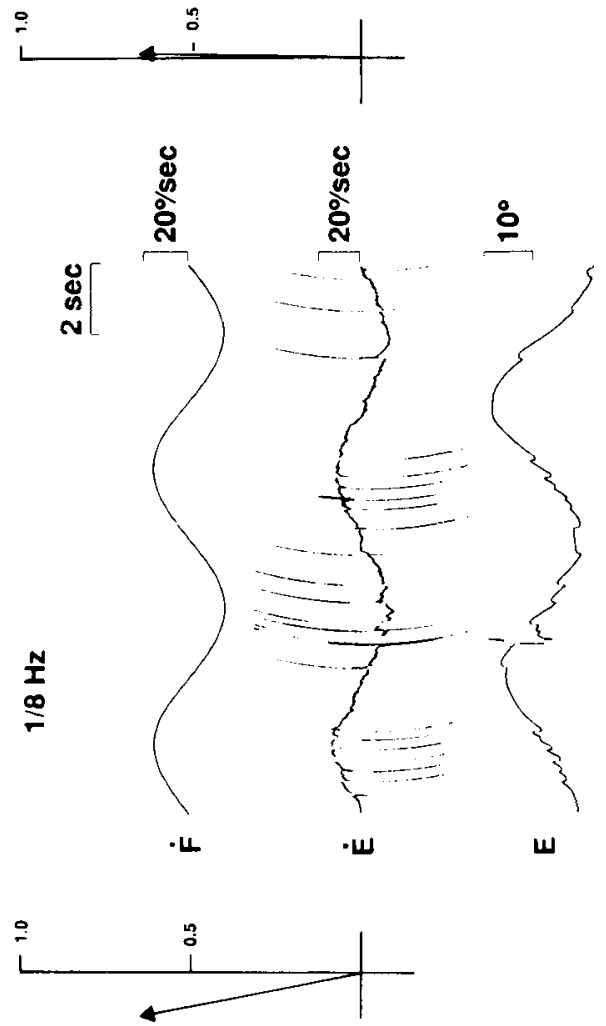

$\sum_{0}^{0}$

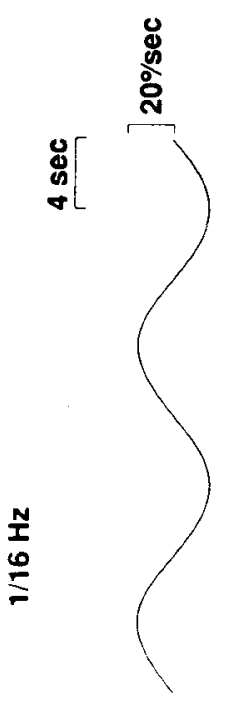

$\cdot 4$
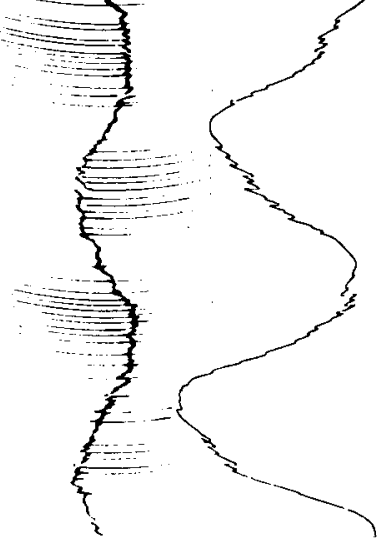

U
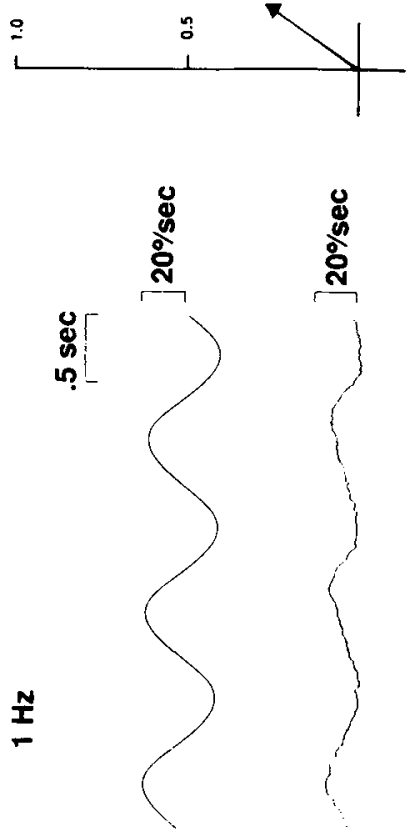

ֻू

$\cdot 4$

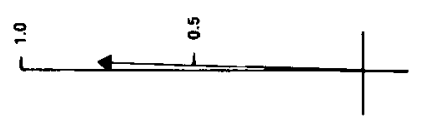<smiles>C1CCC1</smiles>

足
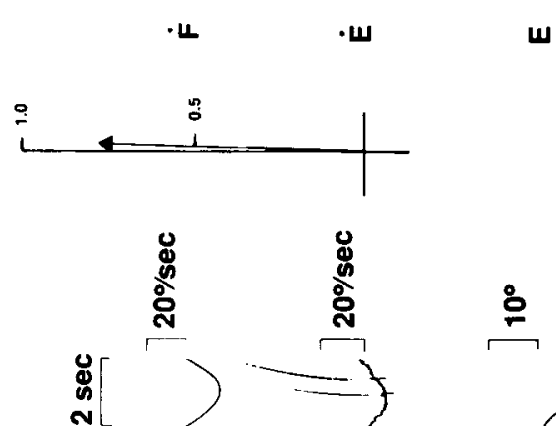

w
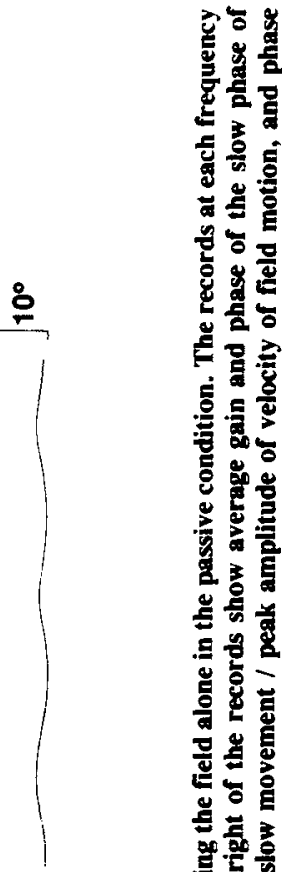

跣

28 은

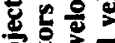

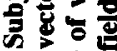

过䒠

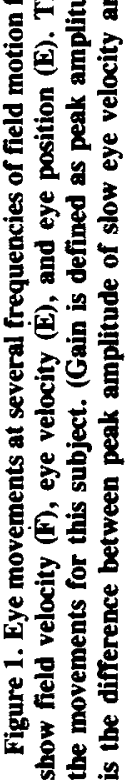



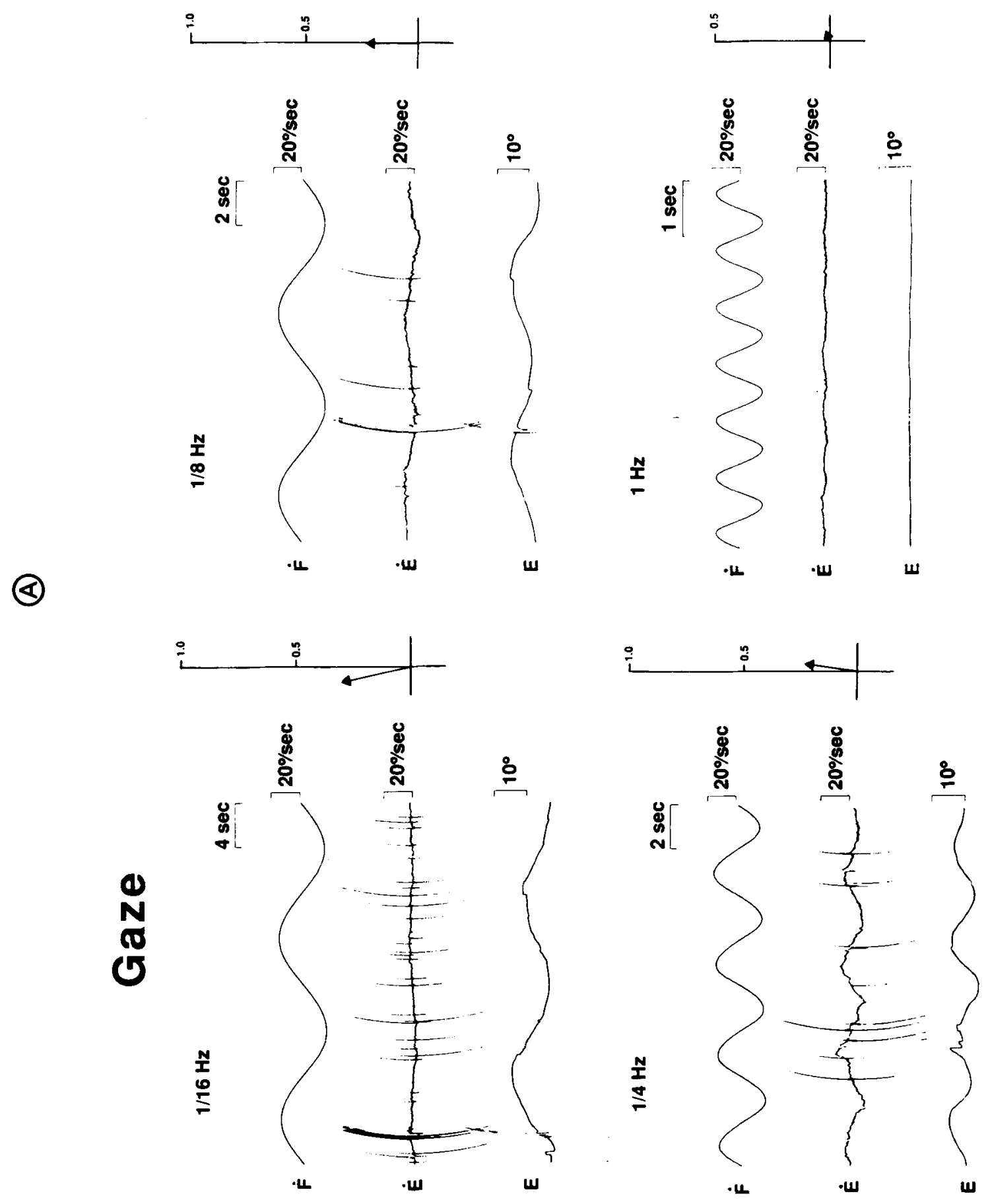


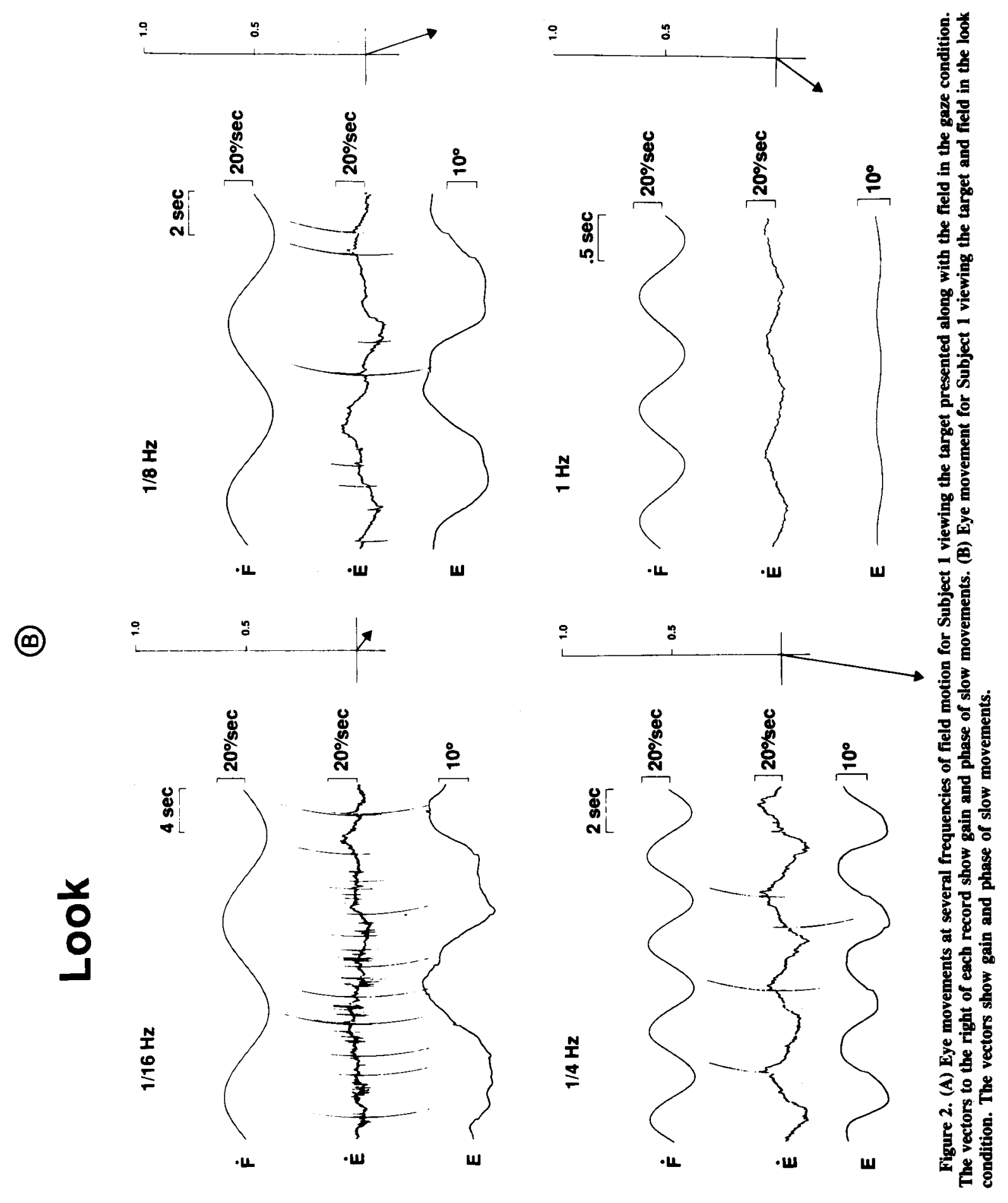



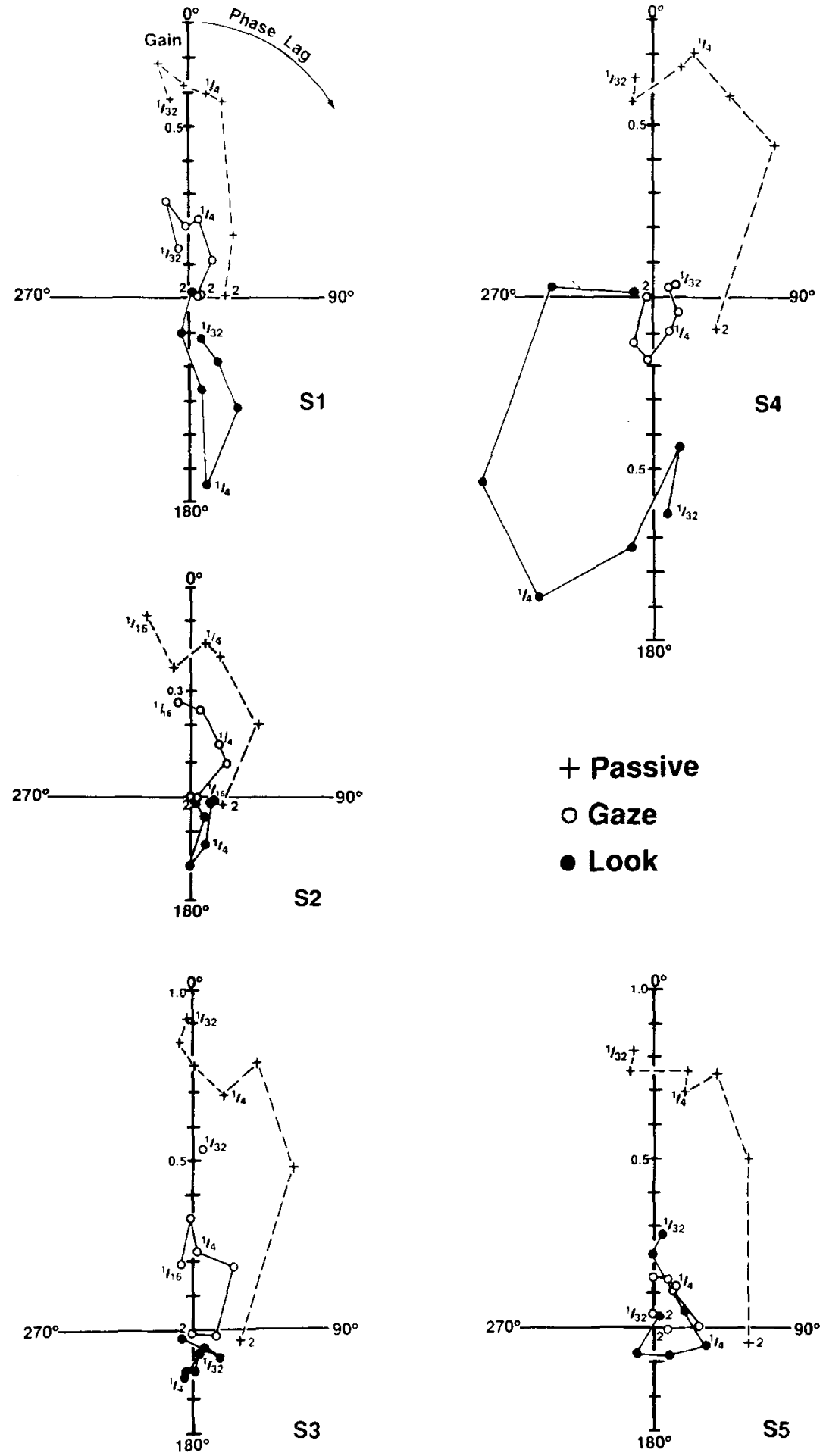

Figure 3. Gain and phase of slow eye movements in the passive, gaze, and look conditions are plotted as a function of frequency on polar coordinates. The phase of the movements in the passive and gaze conditions are about the same, and roughly opposite to the phase in the look condition. Gain standard deviations $(S D s)$ in all three conditions varied from $0.06-0.13$ (peak velocity of about $1^{\circ}-2^{\circ} / \mathrm{sec}$ ), except at $2 \mathrm{~Hz}$, where they varied from 0.02 to 0.04 (about $0.25^{\circ}-0.6^{\circ} / \mathrm{sec}$ ). Phase $S D$ s in the passive condition varied from about $1^{\circ}$ to $5^{\circ}$, except at $2 \mathrm{~Hz}$, where they were somewhat larger. Phase $S D \mathrm{~s}$ in the gaze and look conditions ranged from $20^{\circ}$ to $38^{\circ}$, with a few exceptions. We chose to omit the $S D$ s from the figure for the sake of clarity. 
$p<.001]$. Overall, in the gaze condition, there was substantial suppression of optokinesis across frequency.

In the look condition, the presence of large phase-lag residual movements, including many instances of roughly counterphase movements, is apparent in Figure 3 (filled circles). Perhaps one way to describe these responses is that they are approximately in counterphase with the passive response. (This trend is clearest for $\$ 4$, but there are signs of it for other subjects as well.) Thus, as frequency increased, the phase lag tended to swing from the $90^{\circ}-180^{\circ}$ quadrant to the $180^{\circ}-270^{\circ}$ or even the $270^{\circ}-360^{\circ}$ quadrant. An exception to this was S5, whose look response started in the first quadrant at the lowest frequencies. However, as frequency increased, S5's responses became more like those of the other subjects, swinging rapidly around the origin to larger phase lags, so that the responses were in counterphase with the passive response by $1 \mathrm{~Hz}$.

The gain of the look response was quite variable from one subject to another, some showing quite large responses and others very small responses, in line with our earlier results (Wyatt \& Pola, 1984). Nevertheless, a general tendency of the look response was for the gain to show a local maximum at $1 / 4-1 / 2 \mathrm{~Hz}$, with relatively smaller responses at both higher and lower frequencies. (This was clearest for $S 1, S 2$, and $S 4$, but it may also be seen for S3 and S5.)

In the gaze condition, 1 subject (S4) showed responses that were often approximately in counterphase with the field, which was quite different from the usual pattern (see Figure 3 ). However, this subject made particularly vigorous counterphase responses in the look condition. Thus, if the gaze condition is considered to be intermediate (in the vector sense) between the passive and look conditions, nearly all of the gaze data in Figure 3 fit such a description. ${ }^{1}$

\section{Hold and Hold-No-Target Conditions}

Figure 4 shows some individual responses of 1 subject (S1) in the hold and hold-no-target conditions at $0.25 \mathrm{~Hz}$. It is apparent that there was very little smooth eye movement in the hold condition and thus substantial suppression. On the other hand, there was a great deal of movement in the hold-no-target condition; in fact, the records appear similar to the passive (optokinetic) responses also shown in the figure.

These two features in the hold and hold-no-target conditions were commonly observed over a wide range of frequencies, as may be seen in Figure 5, which shows data plotted on polar coordinates for all 5 subjects. Passive responses from Figure 3 have been repeated in Figure 5 as a reference. The data points for the hold condition (filled squares) are all clustered near the origin, indicating good suppression over all frequencies. (For very small gains, the phase lags must be regarded as essentially arbitrary.) However, at $1 / 2 \mathrm{~Hz}$ and/or $1 \mathrm{~Hz}$, small but distinguishable eye movements occurred for some subjects (S3-S5). (Small, low-frequency responses were also seen for $\mathrm{S} 2$.)
In contrast with responses in the hold condition, the responses in the hold-no-target condition (Figure 5, open squares) approximately parallelled those in the passive condition. A two-way ANOVA performed on the phases in the two conditions showed no effect of instruction $[F(1,4)=2.2, p=.21]$ and no interaction between instruction and frequency $[F(6,24)=.93, p=.49]$. For 3 of the subjects (S1, S3, and S4), the hold-no-target gains were about $0.4-0.6$ times as large as the passive gains, except at 1 and $2 \mathrm{~Hz}$ for $\mathrm{S} 1$ and at $1 / 2$ and $1 \mathrm{~Hz}$ for $\mathrm{S} 3$, when the gains in the two conditions were similar. Two subjects (S2 and S5) showed similar responses at all frequencies in the two conditions (except for $S 2$ at $1 / 16 \mathrm{~Hz}$, when the hold-no-target was distinctly smaller than the passive). It is interesting to note that for nearly every subject and frequency, there was considerably more suppression in the gaze condition (Figure 3 ) than in the hold-notarget condition (Figure 5). In other words, suppression of optokinesis was much stronger with a target but no effort than with effort but no target.

\section{DISCUSSION}

The central finding of this experiment is that a target stabilized at the foveal retina can suppress optokinetic eye movements over a broad range of frequencies, and that the characteristic of this frequency-dependent response can be influenced by instructions given to the subject. These results are generally consistent with those in previous studies (Howard et al., 1989; Wyatt \& Pola, 1984; Wyatt et al., 1988), but they give a profile of the overall dynamics of some of the mechanisms used in suppressing optokinesis.

\section{The Passive Condition: Optokinetic Responses to Sinusoidal Stimulation}

Smooth passive optokinetic responses showed a decrease in gain and increase in phase lag as the frequency of sinusoidal field motion increased, which is similar to the findings in several earlier studies (Pola \& Wyatt, 1985; Wyatt \& Pola, 1984; Wyatt \& Pola, 1988). Little work on optokinesis has been done with sine-wave field motion; however, in one other study of human optokinesis, similar patterns of gain and phase lag were obtained (Bohmer \& Allum, 1981). Similar gains and phases have also been observed with rabbits (Collewijn, 1969, 1972) and with monkeys (Boyle, Buttner, \& Markert, 1985; Paige, 1983). It is interesting that the fairly high gain and small phase lags observed here and in other studies, especially at higher frequencies, appear to depend on predictive capability in the optokinetic system (Wyatt \& Pola, 1988).

\section{Gaze and Look Conditions}

The results in the gaze condition show that when subjects avoid deliberate regard of the stabilized target and field, substantial suppression of optokinesis is found over a broad range of frequencies of field motion. These findings extend our earlier work (Wyatt et al., 1988), in which 

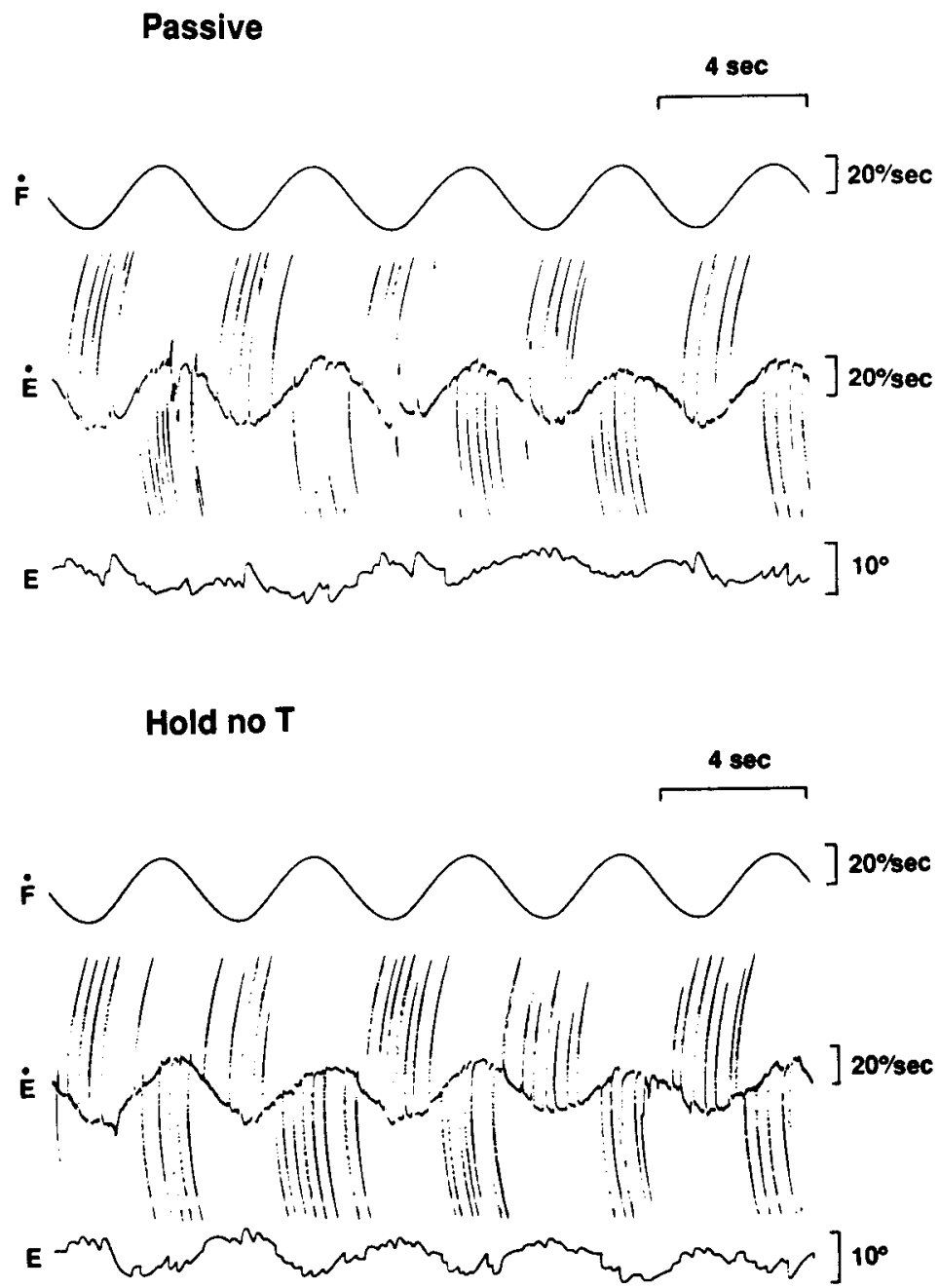

Hold

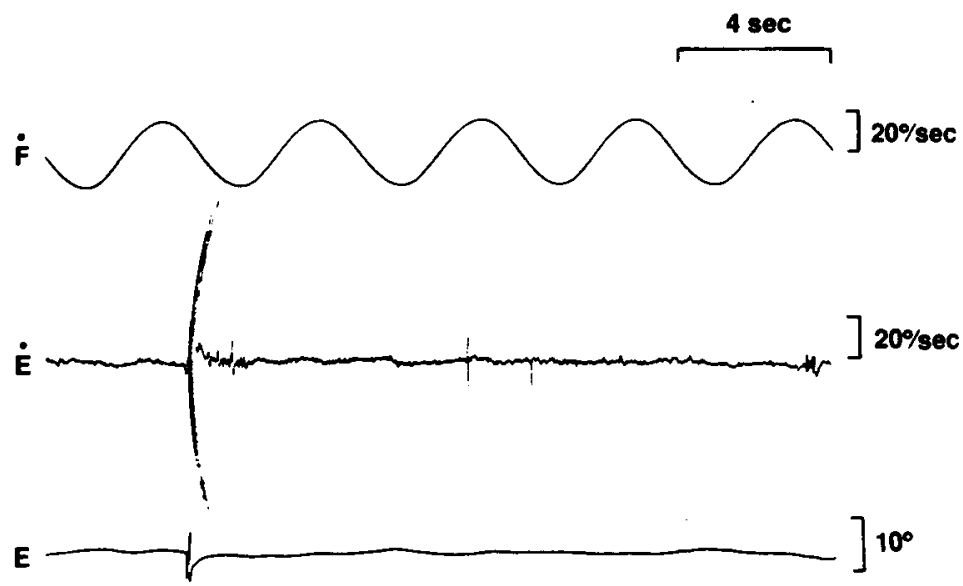

Figure 4. Eye movements at one frequency of field motion for Subject 1 in the passive, hold-no-target, and hold conditions. Note that the passive and hold-notarget responses are about the same, whereas there is little or no movement in the hold condition. 

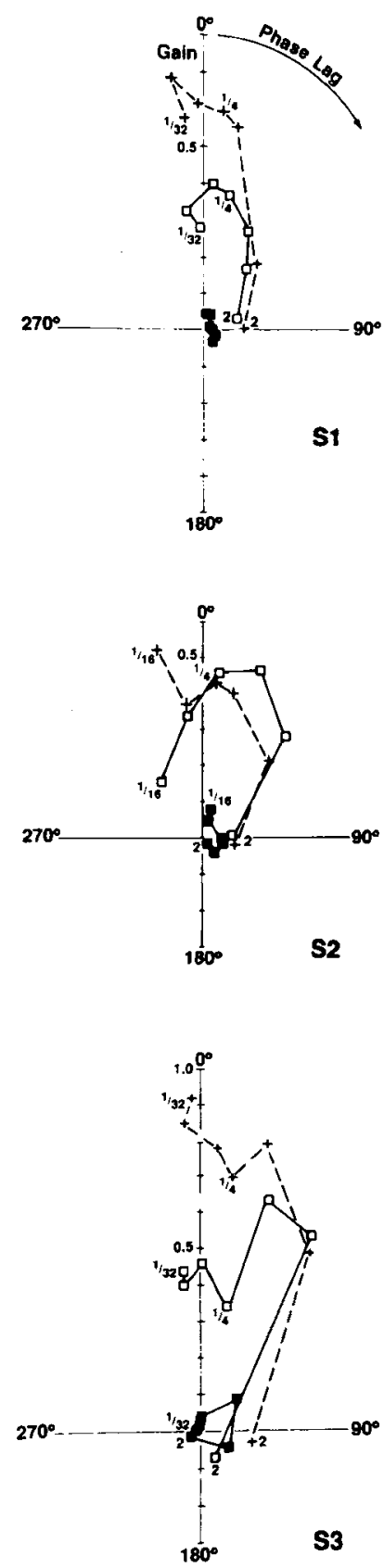

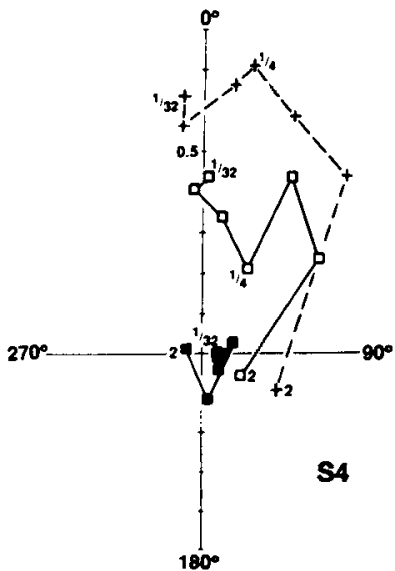

+ Passive a Hold no T

- Hold

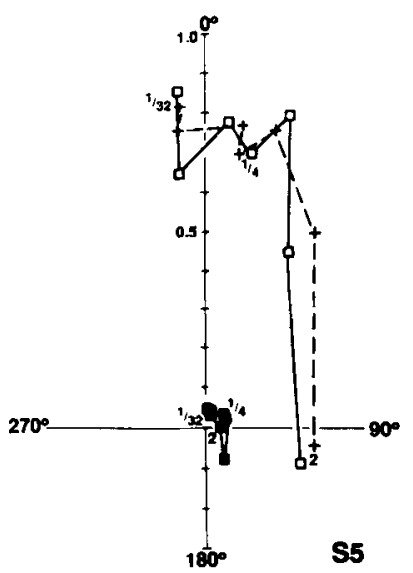

Figure 5. Gain and phase of slow movements in the hold and hold-no-target conditions presented with respect to frequency on polar coordinates. Passive data (Figure 3) are also shown as a reference. In the hold condition, gain standard deviations $(S D \mathrm{~s})$ varied from 0.2 to $0.06\left(0.3^{\circ}-1^{\circ} / \mathrm{sec}\right)$, whereas in the hold-notarget condition, they varied from 0.06 to $0.13\left(1^{\circ}-2^{\circ} / \mathrm{sec}\right)$. Hold phase SDs varied from $20^{\circ}$ to $38^{\circ}$ and hold-no-target phase $S D$ s ranged from $1^{\circ}$ to $5^{\circ}$. 
we have found suppression in the gaze condition (referred to there as "passive suppression") and demonstrated its operation for extrafoveal as well as foveal targets. The fact that gaze suppression with eye movements roughly in phase with field motion occurred at frequencies as low as $1 / 32 \mathrm{~Hz}$ suggests that suppression with similar characteristics should also occur for unidirectional field motion. ${ }^{2}$ We have in fact observed gaze suppression for unidirectional field motion with several subjects (unpublished observations), and there have been several other reports of what appears to be such suppression for unidirectional motion with the use of a stabilized scotoma (Cheng \& Outerbridge, 1975; Dubois \& Collewijn, 1979). In all of these studies, the extent of eye movement was much less than would be the case for field motion alone, and the movement was in the direction of field motion.

The results in the look condition show that when actively looking at a stabilized target, subjects make slow residual eye movements with large phase lags, over a wide range of frequencies of field motion. This confirms and extends our previous work (Wyatt \& Pola, 1984). The phase lag in the look condition was roughly $180^{\circ}$ for all subjects at about $1 / 4 \mathrm{~Hz}$, and it tended to increase at higher frequencies for most subjects. Subject $S 5$ had relatively small phase lag at low frequencies, but did show counterphase movements at $1 / 4 \mathrm{~Hz}$ and above. Other subjects (S1, S3, and S4) showed counterphase-type movements even at the lowest frequency, which suggests that these subjects should respond with slow movements opposite in direction to unidirectional field motion. ${ }^{2}$ This was in fact verified for the 3 subjects (unpublished observations). An interesting feature of the present results is that at midrange frequencies $(1 / 4-1 / 2 \mathrm{~Hz})$ the responses in the look condition show a maximum in gain. A fall-off in gain at high frequencies is found in most physical systems and thus is not surprising here; however, the falloff at low frequencies is not necessarily to be expected. The finding that responses in the look and gaze conditions show consistent differences across frequencies supports the view that the look condition involves processes in addition to those operating in the gaze condition (Wyatt et al., 1988).

As noted in the Results section, it may be more appropriate to describe (a given subject's) eye movements in the look condition as being in counterphase with (the subject's) eye movements in the passive condition, rather than in counterphase with the field. Another feature of the present results is that (with certain exceptions noted in the Results section) the passive, gaze, and look conditions seem to form an approximate continuum, with gaze representing an intermediate position between the passive and look conditions. This is a continuum in the vector sense. Gaze responses tend to fall on or near the line joining the passive and look data points for the same frequency and subject. (Since the look response is usually approximately in counterphase with the passive response for a given subject, the line joining the two points passes through or near the origin.)
One study (Mack, Fendrich, \& Wong, 1982) did not yield residual movements in circumstances similar to our look condition; however, we suspect (Wyatt \& Pola, 1984) that those results may have depended on a very restricted stimulus field in comparison with the "world-like" optokinetic field of the present experiments. Collewijn and Tamminga (1986) recently conducted experiments with subjects regarding a stabilized target presented against periodic background motion. They found suppression of optokinesis with residual smooth eye movements having substantial phase lag, but the movements were very idiosyncratic. They argued that such variability called the technique into question. However, it is important to note that their experimental situation involved very-smallamplitude field motion $\left(1^{\circ}-4^{\circ}\right.$ peak to peak $)$ and also sumof-sines stimuli, which are known to produce responses both complex and not well understood (Barnes \& Ruddock, 1989; Wyatt \& Pola, 1988).

It has been known for some time that with deliberate effort a subject viewing a foveally stabilized target or afterimage can create or modify smooth eye movements (Heywood, 1972; Heywood \& Churcher, 1971; Mack \& Bachant, 1969; Steinbach \& Pearce, 1972). A recent demonstration of this, relevant to the present work, is that subjects can influence whether a stabilized target moves in phase with or in counterphase with a field moving sinusoidally (van den Berg \& Collewijn, 1987). This ability may be related to forcing a mental set of whether the target is moving with or opposite to the field (van den Berg \& Collewijn, 1987), but it may also come from the ability to attend to and use a restricted (extrafoveal) portion of the target to generate smooth pursuit eye movements (i.e., using target position as stimulus; see Pola \& Wyatt, 1980). One reason we used a target that was larger than usual $\left(4^{\circ}\right)$ was that it reduced contours in the immediate vicinity of the fovea-contours that we felt might exacerbate idosyncratic drifts and movements. In any case, it should be noted that voluntary control is not unique to slow eye movements with stabilized targets, but that it is a factor that must be confronted with virtually all eye movements under many experimental circumstances. For example, subjects can voluntarily modulate the velocity of smooth pursuit movements (Steinman, Skavenski, \& Sanbury, 1969), modify the vestibulo-ocular reflex (Barr, Schultheis, \& Robinson, 1976), and regulate the occurrence of saccadic eye movements during pursuit of a moving target (Puckett \& Steinman, 1969) or fixation of a stationary target (Steinman, Cunitz, Timberlake, \& Herman, 1967).

In the present experiments, we were careful to instruct subjects about how to regard the target on a given trial, with the intent of minimizing the intrusion of undesirable voluntary efforts. Especially in the look condition, we made it clear that subjects were not in any way to attempt to voluntarily manipulate target motion. ${ }^{3}$ Instructions to simply look at the target, as well as our other instructions, gave results that were reliable within and across subjects (e.g., see the standard deviations in Figures 3 and 5), and 


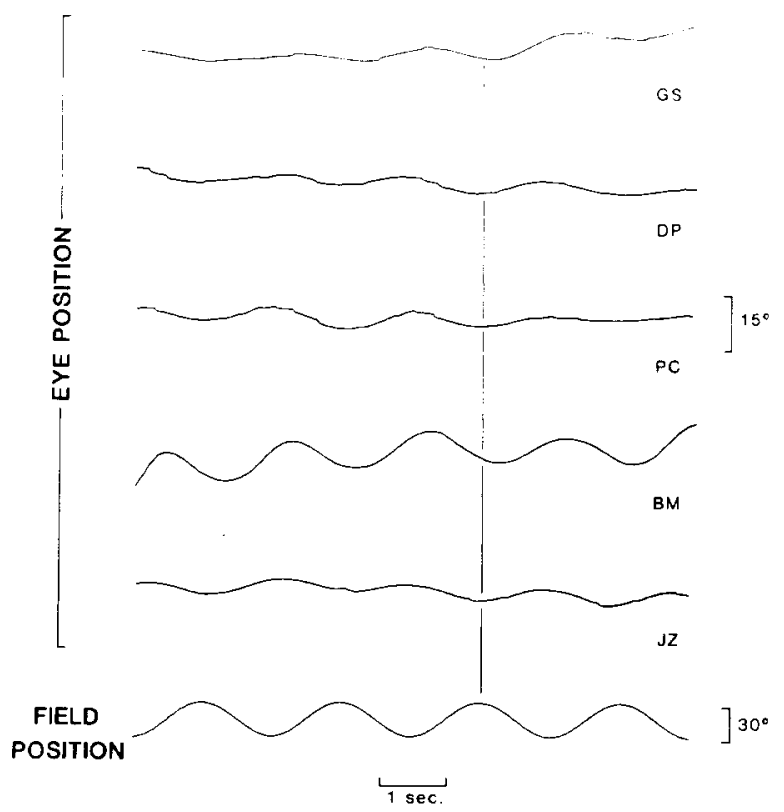

Figure 6. First-trial-ever responses of 5 naive subjects in the look condition. All subjects show clear counterphase movements.

the subjects reported no difficulty in carrying out the tasks (except, possibly, the hold tasks). (Individual tendencies to fixate a point slightly off target center or to drift in one or another direction were compensated for by slight offset of the target; the use of a largish target in the present work helped to obviate difficulties that can arise if a very small target moves slightly off the fovea.)

To illustrate of the generality of look responses, Figure 6 presents records from 5 first-trial-ever naive subjects in the look condition, who were instructed as in the Method section; eye movements are approximately in counterphase with field motion at $1 / 2 \mathrm{~Hz}$. (These 5 subjects were additional to those in the present paper.) In a recent study done with the look condition (Lustgarten, Pola, \& Wyatt, 1988), suppression of optokinesis in the stabilized condition was compared with suppression in the normal closed-loop condition. The main finding was that small-amplitude eye movements, with phase lags of about $90^{\circ}-180^{\circ}$, persisted in the closed-loop condition. Moreover, these movements could be accurately predicted from the counterphase-type movements found in the stabilized condition. It should be noted that Tamminga and Collewijn (1981) observed similar residual movements in the closedloop condition. This indicates that the oculomotor response occurring in the look condition is not restricted to subjects viewing a stabilized target, and that a related response occurs under normal viewing conditions. ${ }^{4}$

\section{Hold and Hold-No-Target Conditions}

In the gaze and look conditions, the task required little effort, with the subject either not trying to do anything in particular or simply looking at a target. Both conditions produced substantial suppression of optokinesis but with residual movements, roughly in phase with or in counterphase with field motion. The hold condition was introduced to see whether or not deliberate effort could suppress optokinesis further, and clearly it could: residual movements at all frequencies ranged from being very small to being nonexistent. (An interesting side note is that the finding that passive, gaze, and look responses often fall on a line through the origin suggests that a condition between gaze and look-or gaze and passive for S4-would also result in near-zero eye movement.) However, the hold-no-target results make it clear that attention and effort alone are not adequate to suppress optokinesis more than modestly; both the presence of a target and attention are important. ${ }^{5}$ It should be noted that this conclusion applies when a subject's gaze is directed at the level of the optokinetic field. Howard and Gonzalez (1987) found substantial suppression of optokinesis if subjects gazed at a location in front of an optokinetic field, and Jung and Kornhuber (1964) found some suppression if subjects gazed into the distance behind a field.

We have not tried varying instruction in association with an extrafoveal target. Barnes and Crombie (1985) had subjects suppress optokinesis by fixating between a pair of stationary (closed-loop) targets; as might be expected, suppression decreased as target separation increased. Attending to an eccentric stationary target would presumably give at least as much suppression as would the gaze condition with an eccentric stabilized target, for which suppression is substantial (Wyatt et al., 1988). (However, our target may have been more salient than that of Barnes and Crombie.) A direct comparison of results with various conditions and targets would be necessary for testing how well all of these results fit together.

\section{The Mechanism of Suppression of Optokinesis With Stabilized Targets}

There are at least two accounts of suppression of optokinesis with stabilized targets; in one, the simple presence of a target switches off optokinesis, and in the other, an active process is involved in eliminating the optokinetic response. Fletcher, Hain, and Zee (1987) found that afternystagmus was absent after subjects looked at a fixed target during optokinetic stimulation, which provides some support for the first possibility. On the other hand, our finding that the overall frequency characteristics of passive, gaze, and look responses (Figure 3) tend to form a continuum (with the look response roughly opposite to the passive) suggests that more is involved than a switch or a volume control.

As well as suggesting an active process, the passive/ gaze/look relationship suggests that the residual movements arise in part from modulation of the optokinetic response. Thus, gaze responses, with smaller gains than, but similar phase lags to, those of passive responses, may be residual optokinetic movements. Look responses, often in counterphase with the passive, may come from a process involving a signal that is in counterphase and that interacts with optokinesis. In the hold condition, although there was little or no residual movement, there was still evidence for a dynamic process, in that subjects reported that effort was required. Residual eye movements may 
involve prediction, since optokinetic movements seem to involve a predictive component (Wyatt \& Pola, 1988). In fact, recent work indicates that residual movements in the look condition are largely the result of a predictive mechanism (Aksionoff, Wyatt, Pola, \& Lustgarten, 1989).

What stimuli regulate the various modes of suppressive behavior? In the gaze condition, the subjects did not try to do anything in particular, and thus their oculomotor responses presumably depended largely on the external stimuli-in particular, on relative motion between target and field (Wyatt et al., 1988). In the look condition, looking at (attending to) a foveally stabilized target might enhance the activity of relative-motion mechanisms, enhancing suppression and perhaps providing a signal for counterphase movements. (It is worth noting that what is called attentional enhancement of neuronal activity occurs commonly in the visual system; see Bushnell, Goldberg, \& Robinson, 1981; Goldburg \& Wurtz, 1972; Wurtz \& Mohler, 1976.) In the hold condition, the subjects attempted to maintain the target in front of their heads. Success in this task appears to require the use of an extraretinal signal of eye position relative to the head. To explain results in a similar condition, Howard et al. (1989) postulated an extraretinal signal, arguing that without one there would be no indication of when the eyes are actually stationary. Though such a signal seems to be required in the hold condition, in which the eyes did remain relatively stationary, residual movements in the gaze and look conditions suggest that extraretinal signaling may play a much smaller role in such cases (perhaps none at all in subjects like S1 and S4). Overall, it seems that suppression may be substantially determined by target-field relative motion in all conditions, and enhanced by the voluntary acts of looking and/or holding.

The smooth pursuit system is often thought to be a primary mechanism that allows one to remain on target against the influence of the motion of an optokinetic field. Perhaps the most important stimulus for smooth pursuit is retinal slip of a moving target. Attending to the target also seems to be necessary for substantial pursuit to occur (Pola \& Wyatt, 1985; Wyatt \& Pola, 1987). In the gaze, look, and hold conditions, there was no retinal slip, yet substantial suppression of optokinesis occurred. Furthermore, in the gaze condition, the subjects paid little or no attention to the target. In fact, in previous work with the gaze condition (Wyatt et al., 1988), we found that a target stabilized as much as $20^{\circ}$ eccentric to the fovea could suppress optokinesis to a significant degree. These results cannot easily be accounted for in terms of a foveal smooth pursuit system, at least as far as the pursuit system is currently understood to function (i.e., as being dependent on retinal slip and attention). Smooth pursuit would seem to be involved only if it were responsive to relative target-field motion as a stimulus (which is a possibility). Some sort of foveal fixation or stabilization system (Robinson, 1981) could be involved in the suppression, but again, relative motion would have to be a stimulus. Thus, the relation of the present results to current models of the oculomotor system is unclear.

\section{Suppression of Optokinesis in the Real World}

To what extent do the processes studied in these experiments contribute to suppression in the real world? The range of frequencies over which experimental suppression is found, and the rapidity and ease with which it is evoked, suggests that similar suppressive responses occur in a wide variety of natural conditions (e.g., when one is looking at a target fixed in space). What, then, is the role of retinal slip? One possibility is that in the look condition, for example, retinal slip might serve to reduce or eliminate residual movements. In this view, target-field relative motion and the act of looking are responsible for much of the suppression, and retinal slip serves to fine tune the suppression-an important, although supplementary, role. In line with this, we recently found that the gradual introduction of feedback in the look condition caused a systematic decrease in amplitude and phase lag of the residual eye movements, with small but distinct movements remaining in the closed loop (Lustgarten et al., 1988). (As noted above, similar small movements with a closed-loop target were mentioned by Collewijn \& Tamminga, 1986.) This response to retinal slip may well come from the smooth pursuit or perhaps a fixation system.

The role that the different instructional conditions play in the real world, although speculative at this point, is worth considering. Under most circumstances, a person's mode of viewing a target is probably similar to our look condition. This suggests that suppression of optokinesis may often involve residual eye movements opposite to field motion, although substantially reduced by retinal slip. However, in the absence of any active looking at a specific object, relative motion between object and background may partially suppress the optokinetic influence of the background, as in the gaze condition. This could provide rapid preattentive nulling of optokinetic movement. (If the object proved to be of interest, greater suppression could be obtained through looking at it.) Suppression of optokinesis without retinal slip was most effective in the hold condition. A mode of regard similar to this might operate in the real world when one views, say, a large homogeneous object without detail at or near the point of fixation. That is, a signal of eye position in the head could provide a second means, besides retinal slip, of fine tuning the position of the eyes.

\section{REFERENCES}

aksionoff, E. E., Wyatt, H. J., Pola, J., Lustgarten, M. (1989). Does suppression of OKN depend on knowing what the stimulus field will do next? Investigarive Ophthalmology \& Viswal Science, 30(Suppl.), 180.

Bahill, A. T., \& MCDonald, J. D. (1983). Model emulates human smooth pursuit system producing zero latency target tracking. Biological Cybernetics, 48, 213-222.

Barnes, G. R., \& Crombie, J. W. (1985). The interaction of conflicting retinal motion stimuli in oculomotor control. Experimental Brain Research, 59, 548-558.

BARNES, G. R., RudDock, C. J. S. (1989). Factors affecting the predictability of pseudo-random motion stimuli in the pursuit reflex of man. Journal of Physiology, 408, 137-165.

Barr, D. D., Schultheis, L. W., \& Robinson, D. A. (1976). Volun- 
tary, non-visual control of the human vestibulo-ocular reflex. Acto Otolaryngology, 81, 365-375.

Bohmer, A., ALLUM, J. H. J. (1981). Human optokinetic responses under quasi-open and closed loop conditions. Biological Cybernetics, 40, 233-238.

Boyle, R., Buttner, U., \& Markert, G. (1985). Vestibular nuclei activity and eye movements in the aler monkey during sinusoidal optokinetic stimulation. Experimental Brain Research, 57, 362-369.

Bushnell, M. C., GoldberG, M. E., Robinson, D. L. (1981). Behavioral enhancement of visual responses in monkey cerebral cortex: I. Modulation in posterior parietal cortex related to selective visual attention. Journal of Neurophysiology, 46, 755-772.

Cheng, M., \& Outerbridge, J. S. (1975). Optokinetic nystagmus during selective retinal stimulation. Experimental Brain Research, 23, 129-139.

CollewIJ, H. (1969). Optokinetic eye movements in the rabbit: Inputoutput relations. Vision Research, 9, 117-132.

CollewiJn, H. (1972). An analog model of the rabbit's optokinetic system. Brain Research, 36, 71-88

Collewisn, H., Tamminga, E. P. (1986). Human fixation and pursuit in normal and open-loop conditions: Effects of central and peripheral retinal targets. Journal of Physiology, 379, 109-129.

Davies, D. R., \& Parasuraman, R. (1982). The psychology of vigilance. New York: Academic Press.

Dubors, M. F. W., \& Collewis, , H. (1979). Optokinetic reactions in man elicited by localized retinal motion stimuli. Vision Research, 19, $1105-1115$.

Fletcher, W. A., Hain, T. C., \& Zee, D. C. (1987). Steady-state and dynamic responses of the human optokinetic system. Investigative Ophthalmology \& Visual Science, 28(Suppl.), 332.

GoldBe RG, M., WURTz, R. H. (1972). Activity of superior colliculus in behaving monkey: II. Effect of attention on neuronal responses. Journal of Neurophysiology, 35, 560-574.

Gresty, M. , HalmaGyi, M. (1979). Following eye movements in the absence of central vision. Acta Otolaryngology, 87, 477-483

HEYWOOD, S. (1972). Voluntary control of smooth eye movements and their velocity. Nature, 238, 408-410.

Heywood, S., Churcher, J. (1971). Eye movements and the afterimage: I. Tracking the afterimage. Vision Research, 11, 1163-1168.

Howard, I. P., Giaschi, D., \&urasugi, C. M. (1989). Suppression of OKN and VOR by afterimages and imaginary objects. Experimental Brain Research, 75, 139-145.

Howard, I. P., \& Gonza Lez, E. G. (1987). Human optokinetic nystagmus in response to moving binocularly disparate stimuli. Vision Research, 27, 1807-1816.

Jung, R., \& Kornhuber, H. H. (1964). Results of electronystagmography in man: The value of optokinetic, vestibular and spontaneous nystagmus for neurologic diagnosis and research. In M. B. Bender (Ed.), The oculomotor system (pp. 428-488). New York: Harper \& Row.

KoERnER, F., \& SCHILler, P. H. (1972). The optokinetic response under open and closed loop conditions in the monkey. Experimental Brain Research, 14, 318-417.

Lustgarten, M., Pola, J., \& Wyatt, H. J. (1988). "Retinal slip"What is its role in the suppression of optokinesis? Investigative Ophthalmology \& Visual Science, 29(Suppl.), 166.

Mack, A., \& BaChant, J. (1969). Perceived movement of the afterimage during eye movements. Perception \& Psychophysics, 6, 379-384.

Mack, A., Fendrich, R., \& Wong, E. (1982). Is perceived motion a stimulus for smooth pursuit? Vision Research, 22, 77-88.

Murasugi, C. M., Howard, I. P., \& OHMI, M. (1986). Optokinetic nystagmus: The effects of stationary edges, alone and in combination with central occlusion. Vision Research, 26, 1155-1162.

Murasugi, C. M. . Howard, I. P., \& OHMI, M. (1989). Human optokinetic nystagmus: Competition between stationary and moving displays. Perception \& Psychophysics, 45, 137-144.

PAIGE, G. D. (1983). Vestibuloocular reflex and its interactions with visual following mechanisms in the squirrel monkey: I. Response characteristics in normal animals. Journal of Neurophysiology, 49, 134-151.

Pola, J., \& Wyatt, H. W. (1980). Target position and velocity: The stimuli for smooth pursuit eye movements. Vision Research, 20(Suppl.), 523-534

Pola, J., \& Wyatt, H. W. (1985). Active and passive smooth eye movements: Effects of stimulus size and location. Vision Research, 25 , 1063-1076

Posner, M. I., Cohen, Y., \& Rafal, R. D. (1982), Neural system control of spatial orienting. Philosophical Transactions of the Royal Society: Series B, 298, 187-198.

Pucketr, J. de W., Steinman, R. M. (1969). Tracking eye movements with and without saccadic corrections. Vision Research, 9. 695-703.

Robinson, D. A. (1981). Control of eye movements. In J. M. Brookhart, V. B. Mountcastle, V. B. Brooks, \& S. B. Geiger (Eds.), Handbook of physiology: Sec. 1. The nervous system: Vol. 2. Motor control (Pt. 2, pp. 1275-1320). Bethesda, MD: American Physiological Society

Steingach, M. J., \& Pearce, D. C. (1972). Release of pursuit eye movements using after-images. Vision Research, 12, 1307-1311.

Steinman, R. M., Cunitz, G. T., Timberlake, G. T., Herman, M. (1967). Voluntary control of microsaccades during maintained monocular fixation. Science, 155, 1577-1579.

Steinman, R. M., Skavenski, A. A., \&anbury, R. V. (1969). Voluntary control of smooth pursuit velocity. Vision Research, 9, 1167-1171.

Tamminga, G. A., \& Collewin, H. (1981). The effect of a structured background on human oculomotor pursuit of visual targets. Documenta Ophthalmologica Praceedings Series, 30, 668-683.

Treisman, A. (1988). Features and objects: The Fourteenth Barlett Memorial Lecture. Quarterly Joumal of Experimental Psychology, 40A, 201-237

VAN Den Berg, A. V., Collewijn, H. (1987). Voluntary smooth eye movements with foveally stabilized targets. Experimental Brain Research, 68, 195-204.

vaN Die, G., Collewis, H. (1982). Optokinetic nystagmus in man. Human Neurobiology, 1, 111-119.

WURTZ, R. H., MOHLER, C. W. (1976). Enhancement of visual responses in monkey striate cortex and frontal eye fields. Journal of Neurophysiology, 39, 766-772.

WYATT, H. W., \& POLA, J. (1984). A mechanism for suppression of optokinesis. Vision Research, 24, 1931-1945.

WYATT, H. W., PoLA, J. (1987). Smooth eye movements with stepramp stimuli: The influence of attention and stimulus extent. Vision Research, 27, 1565-1580.

Wyatt, H. W., \&ola, J. (1988). Predictive behavior of optokinetic eye movements. Experimental Brain Research, 73, 615-626.

Wyatt, H. W., \& Pola, J., \& Lustgarten, M. (1988). "Passive suppression" of optokinesis by stabilized targets. Vision Research, 28 , 1028-1029.

\section{NOTES}

1. The characteristics of the results could not be due to spatial masking effects of the $4^{\circ}$ target, given that we obtained essentially the same results with a $4^{\circ}$ annulus (which involves essentially no masking). Also, we obtained the same type of results with a $1.5^{\circ}$ target in earlier work (Wyatt \& Pola, 1984, 1988).

2. Unidirectional motion can be regarded as a low-frequency limit, and from this perspective, one would expect that whatever system behavior is found at relatively low frequencies should also be found for unidirectional motion.

3. One possibility that should be considered is that, in the look condition, the subject's locus of attention might reflexively shift from one side of the target to the other at the same frequency as, but in counterphase with, the field motion. A consequence of this might be slow counterphase eye movements. If this is so, residual movements would not come directly from mechanisms involved in suppression, but as a consequence of an interaction of perception of motion and attention. This is one among many conceivable explanations for the large phase lag seen in the look condition, but even if this were true, it is quite distinct from the intentional manipulation of target motion in the studies of van den Berg and Collewijn (1987). 
4. Besides instructions, tasks performed by the subject can define conditions. Such an approach is common in work on attention (Davies \& Parasuraman, 1982; Posner, Cohen, \& Rafal, 1982; Treisman, 1988). In our experiments, in the look condition, subjects were instructed to look at the target actively-to attend to it-whereas in the gaze condition, they were instructed to attempt to avoid deliberate attention to the target or field. A task-defined equivalent to the look condition might involve requiring the subject to detect a brief dimming of the target. Similarly, an equivalent to the gaze condition might consist of requiring the subject to detect brief flashes at random locations in the field. We implemented two vigilance tasks of this sort and ran a number of experimental sessions on several of our subjects. (The actual tasks consisted of the detection of occasional longer dimmings or flashes in a regular series of shorter ones.) The responses with such operationally defined conditions were very similar to the same sort of responses, for each subject, that had been found with the use of the look and gaze in structions. This suggests (1) that our instructional conditions are roughly equivalent to simple operationally defined attentional tasks, and (2) that variability from one subject to another is not the result of differences in idiosyncratic interpretation of instructions, but in large measure appears to come from variation, between subjects, in the parameters of ocular control mechanisms.

5. We did not attempt to develop any operationally defined conditions equivalent to the hold and hold-no-target conditions. In a sense, the nature of such trials provides a built-in task, and, partly because of this, the instructions seemed to be relatively unambiguous and less susceptible to individual interpretation.

(Manuscript received February 25, 1991; revision accepted for publication February 18, 1992.) 